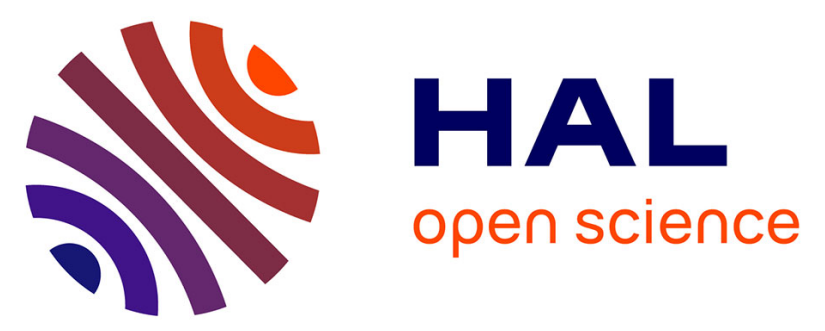

\title{
Ex Vivo bone formation in bovine trabecular bone cultured in a dynamic 3D bioreactor is enhanced by compressive mechanical strain.
}

Valentin David, Alain Guignandon, Aline Martin, Luc Malaval, Marie-Hélène

Lafage-Proust, Aline Rattner, Val Mann, Brendon Noble, David B. Jones, Laurence Vico

\section{To cite this version:}

Valentin David, Alain Guignandon, Aline Martin, Luc Malaval, Marie-Hélène Lafage-Proust, et al.. Ex Vivo bone formation in bovine trabecular bone cultured in a dynamic 3D bioreactor is enhanced by compressive mechanical strain.. Tissue Engineering: Parts A, B, and C, 2008, 14 (1), pp.117-26. 10.1089/ten.a.2007.0051 . ujm-00270273

\section{HAL Id: ujm-00270273}

\section{https://hal-ujm.archives-ouvertes.fr/ujm-00270273}

Submitted on 4 Apr 2008

HAL is a multi-disciplinary open access archive for the deposit and dissemination of scientific research documents, whether they are published or not. The documents may come from teaching and research institutions in France or abroad, or from public or private research centers.
L'archive ouverte pluridisciplinaire $\mathbf{H A L}$, est destinée au dépôt et à la diffusion de documents scientifiques de niveau recherche, publiés ou non, émanant des établissements d'enseignement et de recherche français ou étrangers, des laboratoires publics ou privés. 

mechanical strain

\author{
Authors Valentin David 1,\#,\$, Ph. D. \\ Alain Guignandon 1, \$, Ph. D. \\ Aline Martin $1, \#$, Ph. D. \\ Luc Malaval ${ }^{1}$, Ph. D. \\ Marie-Hélène Lafage-Proust ${ }^{1}$, M.D., Ph. D. \\ Aline Rattner ${ }^{1}$, Ph. D. \\ Val Mann2, Ph. D. \\ Brendon Noble ${ }^{2}$, Ph. D. \\ David B J ones ${ }^{3}$, Ph. D. \\ Laurence Vicol, Ph. D. \\ \$ V.D. and A.G. contributed equally to the work
}

\title{
Affiliations
}

1 - INSERM U890, St Etienne, F-42023, France; Laboratoire de Biologie du Tissu Osseux, Université J ean Monnet, St Etienne, F-42023, France

2 - Scottish Mechanotransduction Consortium, University of Edinburgh, Medical School, Edinburgh, United Kingdom

3 - Experimental Orthopaedics and Biomechanics, Philipps University, Baldingerst, D35033 Marburg, Germany

\# Present address: The Kidney Institute, University of Kansas Medical Center, Kansas City, KS 66160, USA

Funding sources European Space Agency, 14232/NL/SH (CCN3)

Microgravity Programme AO-99-122/ 14426 and Institut National de la Santé et de la Recherche Médicale

Running title Cancellous bone culture in a dynamic 3D bioreactor

\section{Corresponding author}

Alain Guignandon, PhD

INSERM E366, Laboratoire de Biologie du Tissu Osseux,

15 rue Ambroise Paré, F-42023 St Etienne cedex 2,

Phone : 33-477421444, Fax : 33-477575572

Email: Alain.Guignandon@univ-st-etienne.fr

$\begin{array}{ll}\text { Number of Words } & 4028-26876 \text { characters with spaces - } 17 \text { pages } \\ \text { Figures and } & 4 \text { (all black and white) } \\ \text { Tables } & 2\end{array}$

All the authors have no conflicts of interest. 


\section{Abstract}

Our aim was to test cell and trabecular responses to mechanical loading in vitro in a tissue bone explant culture model. The ZetOS ${ }^{\mathrm{TM}}$ system provides the ability to exert cyclic compression on cancellous bone cylinders (bovine sternum) cultured in forced flow circumfusion chambers, and allows assessing mechanical parameters of the cultivated samples. We evaluated bone cellular parameters through osteocyte viability test, gene and protein expression and histomorphometric bone formation rate, in non-loaded versus loaded samples. The microarchitecture of bone cores was appraised by in vivo micro-CT imaging. After 3 weeks, the samples receiving daily cyclic compression exhibited increased osteoblast differentiation and activity associated with thicker, more plate-like shaped trabeculae, higher Young's Modulus and ultimate force as compared to unloaded samples. Osteoclast activity was not affected by mechanical strain, although it was responsive to drug treatments (retinoic acid and bisphosphonate) during the first 2 weeks of culture. Thus, in the ZetOSTM apparatus, we reproduce in vitro the osteogenic effects of mechanical strain known in vivo, making this system a unique and an essential laboratory aid for ex vivo testing of lamellar bone remodelling.

Keywords: trabecular tissue culture; osteoblast, osteoclast; mechanical strain; Young's Modulus 


\section{Introduction}

To better explore local tissue responses to chemical or physical factors, in vitro systems filling the gap between 2D cell culture and a living organism are required. The maintenance or growth of a tissue in culture is delicate, mainly for bone tissue. Up to now, bone organ culture has been performed for a few hours in rat ulnae ${ }^{1},{ }^{2}$ and a few days in embryonic, fœtal or neonatal bone ${ }^{3},{ }^{4},{ }^{5}$. The crucial problem for bone tissue maintenance is ensuring uniform medium diffusion into the structure as well as establishing a mechanically loaded environment. For such in vitro experimentation to truly permit analysis of in situ bone response, away from systemic regulations, the biological requirements would be the following: (1) use cancellous bone which is metabolically more active than cortical bone, (2) with harversian organization similar to human bone, (3) permitting not only cellular responses but also resultant tissue responses, requiring longer term culture, (4) including a loading system, unloading being a cause of disuse.

An ex vivo, 3D explant culture model has been developed in which responses to hydrostatic pressure have been assessed on calve bone cores cleaned of bone marrow ${ }^{6}$. This model did not provide medium perfusion of cultured samples so that analyses were done on the surface of the samples after seeding with osteoblasts. The authors showed that dynamic hydrostatic pressure keeps osteocytes alive and enhances osteoblast function through osteocyte-osteoblast interactions at least during the first 8 days ${ }^{7}$.

A new highly accurate mechanical loading system combined with a cancellous bone diffusion culture-loading chamber has been built ${ }^{8}$, which provides the ability to study bone tissue under constant perfusion and loading conditions. The device, named ZetOSTM, also allows accurate measurement of visco-elastic properties of the tissue. It has been used recently by some of us ${ }^{9}$, who showed that cyclic loading applied to human femoral head cores reduced osteocyte apoptosis and enhanced some osteoblastic functions, in line with the Takai et al results ${ }^{7}$. The goal of the present study was to 
evaluate not only bone cellular responses (osteocytes, osteoblasts, and osteoclasts) to a physiological load stimulus but also bone tissue responses, and to test the bone response to drugs able to modulate bone remodeling in bovine bone samples. 


\section{Materials and Methods}

\section{The Zetos ${ }^{\text {TM }}$ dynamic bioreactor}

The Zetos ${ }^{T M}$ apparatus (Fig. 1A), described in ${ }^{8}$, is kept in a room at $37^{\circ} \mathrm{C}$. The culture chambers (Fig. 1B) are perfused with a peristaltic pump connected to a culture medium tank. For mechanical stimulation, the chambers are introduced in a loading device (see below).

\section{Culture conditions}

The samples (topographic image Fig. 1C) were prepared from bovine bone, which has been successfully used in xenografts to repair bony defects, and has the same characteristics as human bone including a true lamellar organization ${ }^{10}$ (Fig. 1D). The requirements for optimal sample preparation as described in ${ }^{11}$ were scrupulously respected. Bovine bone cores were collected from the ulna and sternum of 6-8 months male calves immediately after sacrifice at the local slaughterhouse. Briefly, ten $\mathrm{mm}$ thick transversal sections were cut from the bones with a band saw (Proxxon $\mathrm{GmbH}$, Niersbach, Germany) and the cores (10 mm diameter) were drilled from the sections and cut to a height of $5 \mathrm{~mm}$, in order to obtain perfectly smooth parallel surfaces. Throughout the cutting procedure the bone was irrigated with sterile $0.9 \% \mathrm{NaCl}$ at $4{ }^{\circ} \mathrm{C}$ in order to limit heat-generated damage, remove bone chips and prevent drying out ${ }^{11}$. Bone cores were washed in Earl's medium + antibiotics (375 IE Streptomycin, IE Penicillin, $4 \mu \mathrm{g}$ Amphotericin B per $1 \mathrm{ml}$ Earle's $1 \mathrm{x}$ ) for $30 \mathrm{~min}$. Bone cores of the basal control group were immediately fixed for histological processing with $4 \%$ formalin; cultured loaded and non loaded cores were fitted in culture chambers, close-fitting in size, filled up with culture medium (DMEM, 10\% FCS, 10-8M ascorbic acid). The assembled system was left to settle for 24 hours at $37^{\circ} \mathrm{C}$ before the start of perfusion (Fig. 1A). Medium was changed every other day and stored for CTX, Tissue Non-specific Alkaline Phosphatase (TNAP) and Calcium assessment. Maximal culture time was 21 days. At the end of the 
culture, the bone cores were fixed in $4 \%$ formalin solution for histology or processed for protein or mRNA extraction. Three independent experiments on 3 animals were performed and at least 10 samples per bone sites (sternum, ulna) were collected.

\section{Pattern of mechanical signal}

Bone cores were submitted to a mechanical signal mimicking ground reaction forces (vertical axe) observed by a weight sensitive platform during a drop jump ${ }^{12}$. Peak loading was set at $4000 \mu$ Strain ( $20 \mu \mathrm{m}$ displacements) after a $5 \mu \mathrm{m}$ preload to ensure contact. 300 cycles were applied per day, at a $1 \mathrm{~Hz}$ frequency. Samples were either perfused (Control, C) or perfused and loaded (Loaded, L) were cultured for 21 days.

\section{Measurement of mechanical properties}

The loading device has been designed to work in two main modes, either to apply a specific compressive strain to a trabecular bone cylinder or to apply a specific force and measure the resulting deformation. The calibrated procedures implanted in the ZetOS software (see ${ }^{8}$ for details) allowed to measure Young's modulus, visco-elastic properties, fracture toughness and fatigue properties of bone. The loading system consists of a piezoelectric crystal stack whose expansion and contraction are controlled by the level of high voltage. A displacement sensor acts in a feedback loop to control the expansion and to control the force applied. We measured ultimate bone mechanical properties at the end of the experiment.

\section{Serial micro-CT imaging}

Bone cores were imaged in sterile tubes filled up with culture medium (Fig 1C) by a $\mu$-CT scanner (Viva CT 40; Scanco Medical, Basserdorf, Switzerland) at a $10 \mu \mathrm{m}$ isotropic voxel size. Each scanning lasted 45 minutes and the estimated radiation dose was $0.35 \mathrm{~Gy}$. We previously ensured on in vitro osteoblast culture that two runs of imaging with this protocol do not affect cell proliferation nor viability, as assessed by Alamar blue test at successive time points (not shown). The linear attenuation coefficient (Lin. Att. 2) of 
thresholded bone was taken as a measurement of tissue mineral density. The bone volume fraction was calculated by plotting gray voxels (bone fraction) against gray plus black voxels (non-bone objects) (VOX-BV/TV). We further measured trabecular thickness (Tb.Th) ${ }^{13}$, Structure Model Index (SMI, ranging from 0 for a plate, and 3 for a rod) ${ }^{14}$, geometric degree of anisotropy (DA) and Connectivity density (Conn-Dens.) ${ }^{15} 16$.

\section{Histomorphometry}

During the culture period two fluorochrome labellings (12 days apart, the last label 24 hours before harvesting) were made with tetracycline $(45 \mu \mathrm{g} / \mathrm{ml}$ of medium). Each label was performed through 6 hours of perfusion with tetracycline supplemented medium, followed by two rinses. After fixation in $4 \%$ formaldehyde, samples were dehydrated in acetone and embedded in methylmethacrylate. Longitudinal medial slices were cut with a Jung Model K microtome (Carl Zeiss, Heidelberg, Germany). Six $8 \mu \mathrm{m}$ thick sections, were used for modified Goldner staining ${ }^{17}$ (Fig. 1F), $14 \mu \mathrm{m}$ thick sections were used to determine the dynamic indices of bone formation (dLS/BS, double labeled surfaces corrected by bone surfaces in \%, MAR, mineral apposition rate in $\mu \mathrm{m} /$ day, and BFR/BS, bone formation rate corrected by bone surfaces expressed in $\mu \mathrm{m}^{3} / \mu \mathrm{m}^{2} /$ day). MAR was derived from fluorochrome interlabel distances (Fig. 1E). BFR were subsequently obtained from the product of $\mathrm{dLS} / \mathrm{BS}$ and MAR ${ }^{18}$. Six $\mu \mathrm{m}$-thick sections were stained with toluidine blue for determination of resorption parameters (Oc.S/BS, Density of lacunae). Structural parameters were measured using an automatic image analysis system (Biocom software, Explora Nova, La Rochelle, France). Bone cellular and macroscopic parameters were measured on a digitizing tablet (Summasketch; Summagraphics, Paris, France) using a dedicated software designed in our laboratory.

\section{Osteocyte viability assessment}

To evaluate osteocyte viability, $10 \mu \mathrm{m}$ thick cryostat sections of undemineralized bone samples were reacted for the presence of LDH activity ${ }^{19}, 9$. Three median sections for 8 controls and 8 loaded samples were assessed for osteocyte viability. LDH and DAPI 
stained cells were counted in 7 fields per section. Viable osteocytes were identified by the presence of dark blue cytoplasmic granules, and their number expressed as a percent of DAPI stained nuclei (Fig. 2 A, B).

\section{Protein extraction}

Total proteins were extracted by grinding bone cores in $2 \mathrm{~mL}$ lysis buffer/well, containing $100 \mu \mathrm{L} / \mathrm{mL}$ Nonidet 40, $1.8 \mathrm{~g} / \mathrm{L}$ lodoacetamide, $3.5 \mu \mathrm{L} / \mathrm{mL}$ PIC (proteases inhibition cocktail, Sigma, France) and $2 \mu \mathrm{L}$-mercaptoethanol, and stored at $-20^{\circ} \mathrm{C}$. Protein concentrations were assayed with the bicinchoninic acid (BCA) protein assay kit (Pierce).

\section{Alkaline phosphatase and ELI SA assays}

TNALP activity was determined by measuring the hydrolysis of p-nitrophenyl phosphate (PNP-p) based on the Lowry method. The product was quantified after 50 min at $37^{\circ} \mathrm{C}$ by spectrophotometry at $412 \mathrm{~nm}$. Activity was expressed as $\mu \mathrm{mol} \mathrm{Pi} / \mathrm{mg}$ proteins/min when measured in collected media, and as a percent of the mean value of D7 unloaded samples when measured in harvested bone cores.

Sandwich Elisas were designed in our laboratory to quantify Osteocalcin (OC) and Runx2 in protein extracts. For sandwich assay we used rabbit anti-human (ADI, San Antonio, TX, USA) and goat anti-human (TEBU, Le Perray en Yvelines, France) antibodies for Runx2 and rabbit anti-human (ABCAM, Paris, France) and mouse anti-bovine (Biosciences, Le Pont de Claix, France) antibodies for OC. Sandwich complex formation is detected with an TNALP-conjugated antibody (Zymed, Cergy Pontoise, France) revealed by incubation with Sigma fast pNP enzyme substrate. Color intensities are measured at $412 \mathrm{~nm}$ and antigen concentrations are calculated from a calibration curve using serial dilution of an arbitrary sample as standards. CrossLaps ${ }^{\circledR}$, a C-terminal peptide of the type I collagen which is a marker of bone resorption, was assayed in culture media using a specific ELISA kit (CrossLaps ${ }^{\circledR}$ Serum, NBD, Herlev, Denmark). 


\section{Calcium measurement}

Assessment of Ca2+ concentrations were carried out on analytical VITROS Ca slides (Ortho Clinical Diagnostics) starting from collected culture media according to manufacturer's instructions.

\section{RNA extraction and real time PCR}

RNA was extracted from tissue at various time points up to 14 days after the beginning of stimulation, using the Chomzinsky and Sacchi method for tissue. RNAs were reverse-transcribed into single-stranded cDNA using first strand cDNA synthesis kit for RT-PCR (AMV, Roche Diagnostics, Meylan, France) and AMV reverse transcriptase. The reaction was incubated for $60 \mathrm{~min}$ at $42^{\circ} \mathrm{C}$. The single strand cDNA was amplified with a

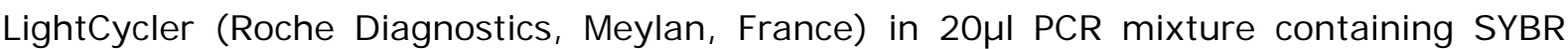
Green I. The fluorescence product was detected at the end of the extension period after $60 \mathrm{~s}$ at $60^{\circ} \mathrm{C}$. Primers used for were for RUNX2: (5') accatggtggagatcatcg, (3') tggggaggatttgtgaagac, product size: 325 bp; for osteocalcin, (5') gcctttgtgtccaagc, (3') ggaccccacatccatag, product size: 315 bp. Quantified data were analyzed with the LightCycler analysis software. The variation in PCR products concentration between experimental groups is expressed as percent of mean control values.

\section{Statistical analysis}

Statistical analysis was performed using the STATISTICA software (StatSoft Inc., Tulsa, OK, USA). One or two way analysis of variance (ANOVA) were performed on protein and RNA data, with the sequentially rejecting Bonferroni-Holm post-test using the Holm's adjusted $\mathrm{p}$-values from the $\mathrm{t}$ table. A factorial analysis was performed on structural bone parameters. Histological, structural/biomechanical and osteocyte viability data were compared with Student's t test after assessing equality of variances and normality of distribution with the Kolmogov-Smirnov test. Results were considered 
significantly different at $p<0.05$. 


\section{Results}

\section{Bone tissue is kept alive and active in the ZetOSTM $^{\text {system. }}$}

The preliminary biological validation procedure of the ZetOS ${ }^{\mathrm{TM}}$ system for a 3-week culture period established that the diffusion rate of nutrients able to maintain bone forming activity was achieved with a $100 \mu \mathrm{l}$ per minute flow. Indeed at this rate, double fluorochrome labelings of mineralizing surfaces were seen throughout ulna and sternum samples (sternum, Fig. 1E). Moreover, the haematopoietic marrow of sternum samples was perfectly preserved during three weeks of culture in the Zetos system (Fig. 1F).

The number of viable osteocytes was lower in control group as compared to loaded group in ulna samples, while it stayed at an acceptable level ( $60 \%$ of viability after 3 weeks) in sternum samples whatever the conditions (Fig. 2C). This result indicated that ulna samples were less suitable for long-term tissue culture. Accordingly, mechanical signals did not affect the bone formation rate (BFR), a direct measurement of new bone forming activity, in ulna samples while it increased the BFR significantly in sternum bone cores (Fig. 2D). The ulna has a highly anisotropic structure (degree of anisotropy, $\mathrm{DA}=2.34 \pm 0.023$, mean $\pm \mathrm{SEM}, \mathrm{n}=10$ ) and load was applied in the long bone axis. The sternum structure is much more isotropic ( $D A=1.58 \pm 0.003$, Table 2). Because of sternum responsiveness to mechanical signals, the rest of this study was performed on samples from sternum bone.

\section{Mechanical strain stimulates osteoblastic activity and bone formation, not bone resorption.}

Alkaline phosphatase activity, an early marker of osteoblastic differentiation, measured either in collected media (Fig 3A) or in tissue lysates (Fig 3B), was stimulated by mechanical strain during the first two weeks of culture. Furthermore, Runx2 protein levels (Fig 3C) and gene expression (Fig 3D) were also higher in loaded samples after 7 and 14 days, and decreased only at day 21 . Mechanical stimulation also increased osteocalcin synthesis after 7 and 14 days (Fig 3E), and its gene expression at day 7 (Fig 
3F). As already shown, histomorphometric data indicated that bone forming activities (maintained in perfused conditions as compared to basal control) were stimulated by cyclic compression (Table 1). This was evidenced by increased osteoid surfaces and thickness and by increased BFR as a consequence of the increase in mineral apposition rate, after three weeks of dynamic culture.

We found that the density of resorption lacunae was significantly reduced in control and loaded conditions as compared to basal controls. Mechanical strain did not alter overall bone resorption since neither histological parameters such as osteoclastic surfaces (Table 1) nor type I collagen C-telopeptide (CTX) levels (Fig 4A) or cathepsin K mRNA expression (data not shown) varied significantly between control and loaded groups. In both control and loaded samples CTX levels suddenly decreased after the first week of culture, then subsequently increased while remaining under day- 6 levels (Fig 4A).

\section{In the ZetOS ${ }^{\mathrm{TM}}$ system, osteoclasts respond to drugs.}

We then asked whether osteoclasts were still responsive in this culture model. Osteoclast stimulation by retinoic acid increased $\mathrm{Ca}^{2}+$ release and/or decreased $\mathrm{Ca}^{2+}$ consumption (Fig 4B) during the entire culture period, although after day $10 \mathrm{Ca}^{2+}$ content of the eluate declined progressively in all groups. Moreover, retinoic acid treatment dramatically increased CTX levels during the first two weeks, and subsequent addition of pamidronate suppressed this osteoclast activation (Fig 4C). Neither retinoic acid nor pamidronate treatment modified phosphate levels and alkaline phosphatase activity (data not shown).

\section{Mechanical strain drives bone samples towards a more resistant architecture.}

As bone forming activity was stimulated and resorption not affected by our mechanical conditions, we speculated that microarchitectural and biomechanical parameters could have been modified by dynamic bioreactor culture. We found (Table 2) that although bone volume was not significantly responsive to mechanical strain, architectural parameters were all significantly increased, resulting in a structure more resistant to 
deformations. Mineral density was not modified. A factorial analysis revealed that architectural parameters SMI and Tb.Th were the most discriminative (not shown). In a further experiment, we performed a follow up assessment of bone quality by $\mu$-CT, with one measurement right after bone core drilling and the other at the end of the 3 weeks culture period. This longitudinal exploration supported our previous observations, confirming trabecular thickening (Fig. 5A) and SMI decrease (Fig. 5B) in loaded samples. These architectural adjustments resulted in an increased Young's modulus under loaded conditions (Fig. 5C) as well as a higher Ultimate Force (Fig. 5D). Although energy of fracture and toughness also tended to increase in loaded samples, the effect did not reach significant levels (not shown). 


\section{Discussion}

When testing any excised specimen of trabecular bone, an unavoidable experimental artefact originates from the sides of the specimen where peripheral trabeculae lose their original load-bearing capacity due to interruption of connectivity. The magnitude of this "side-artefact" errors in modulus measurement has already been assessed in free isolated samples ${ }^{20}$, for which proper mechanical testing requires a specimen size that is greater than $4-5 \mathrm{~mm}$ to satisfy the continuum assumption ${ }^{21}$ but below $10 \mathrm{~mm}$ due to the spatial heterogeneity in volume fraction, micro-architecture, and orientation of trabecular bone. Our sample size was in accordance with these recommendations, but the same error assessment still has to be made for specimen within a close-fitting chamber.

In our first set of experiments, we tested two trabecular bone types originating from the sternum and the ulna. Bone formation rate increased with loading in sternum only, while viable osteocytes decreased with unloading in ulna: this suggests that unloading constitutes a disuse condition for the weight-bearing ulna, but not for the less weightbearing sternum. We thus decided to focus on the adaptative response of sternum for the rest of the study.

We demonstrated in this study that a physiologically relevant mechanical signal favors bone formation in the sternum. Both static and dynamic histomorphometric indices of bone formation were higher in loaded than in unloaded samples. Changes in biological marker expression strengthened these histological features. Indeed, osteogenic markers (alkaline phosphatase, Runx2, osteocalcin) exhibited higher levels of expression than in perfused samples alone. The time course observed for TNALP activity is in agreement with its known temporal expression in vitro, characterized by a peak during the matrix maturation phase and a decrease at later stages $\left({ }^{22},{ }^{23}\right)$. Our study showed that the temporal increase in expression of osteocalcin and runx2 started at an earlier time under loading, indicating that they may be associated with a stimulation of osteoblastic cell differentiation and function by mechanical signals. In contrast, mechanical stimulation 
had no effect on the histological parameters and biological markers (CTX and cathepsin K) of bone resorption. Resorption activity is nonetheless still reactive in this culture system as it is stimulated by a classical enhancing factor (retinoic acid) and inhibited by bisphosphonates. These results showed that in the ZetOSTM system, bone resorption can be challenged by drugs. This model might thus offer an alternative over the widely used rat animal model, whose bone responses to bisphosphonates, in particular, should be interpreted with caution due to the prevalence of modeling over remodeling effects even at the end of the rapid growing phase ${ }^{24}$.

Mechanical-induced altered cellular activities characterized by stimulation of bone formation without change in bone resorption were translated into enhanced bone morphology and strength. Microarchitecture changes included thickening of trabeculae as expected in view of the positive bone remodeling balance, as well as reorganization of the trabecular network towards a more plate-like structure, known to be well suited to bear loads from one defined direction whereas rod-like structures show more flexibility as to the direction of the load ${ }^{25}$. We also showed that microarchitecture alterations were associated with improved bone mechanical competence that reached statistical significance for Young's modulus and ultimate force. Of note, these parameters were unchanged in unloaded samples vs basal controls, confirming that the increase seen in loaded samples was strictly due to the mechanical regimen.

A limitation of this culture system is that, as compared to basal controls, ZetOsTM perfused samples showed less resorption lacunae after 3 weeks of culture, suggesting a reduced number of working osteoclasts, in accordance with the known 2-week osteoclast lifespan ${ }^{26}$. We also noted that Runx2 gene expression is greatly reduced by the end of the culture period suggesting that the osteogenic potential of precursor cells is declining at this time. Our cellular investigations indicated that bone cellular activities are responsive up to a certain extent. Without media perfusion, Takai et al. ${ }^{7}$ estimates that in their explant culture model repopulated with osteoblasts after marrow flushing, the cellular data are robust up to day 8 . Here we demonstrated that the culture time period that we used ( 3 weeks) is compatible with cell and tissue adaptation of a whole bone 
explant, but future studies are needed to gain detailed information on the long term competence of haematopoietic and mesenchymal marrow cells in this system.

The ZetOSTM ex vivo culture model seems to recapitulate in vivo effects of osteogenic exercises. Indeed in both human and animals, it has been demonstrated that osteogenic exercises induce increases in bone formation without affecting bone resorption resulting in thicker trabeculae $\left({ }^{27},{ }^{28},{ }^{29}\right)$. That our ex vivo culture model recapitulates these effects further validates the relevance of the ZetOsTM system. Furthermore, the complex action of mechanical forces in vivo, including rearrangements of trabecular bone architecture and thus changes of mechanical resistance can be mimicked in this culture model.

In conclusion, mechanical loading of ex vivo 3D bone tissue cultures induces bone formation that results in the promotion of appropriate tissue geometry and mechanical competence. This system may be viewed as a new model of preclinical experimentation using bone from large mammals that would allow the evaluation of biomechanical and microarchitectural properties as well as remodelling activities in the cancellous haversian bone compartment. Thus, the ZetOSTM system may be useful for studying bone metabolism in pharmaceutical research, which would reduce the numbers of rodent animal experiments required for product development. It may be also useful for bone tissue engineering, a considerable clinical and scientific issue, with the challenge to provide a bioreactor able to cultivate the cell/scaffold constructs in a perfused and dynamic in vitro environment supporting tissue growth. 


\section{Acknowledgements}

The authors thank Norbert Laroche for his great competence in histology and Sylvie Peyroche for expert technical assistance in cell culture. This study was supported by European Space Agency: European Research In Space and Terrestrial Osteoporosis (ERISTO) contract number $14232 / \mathrm{NL} / \mathrm{SH}$ (CCN3) and Microgravity Application Programme AO-99-122 contract number 14426 and by the Institut National de la Santé et de la Recherche Médicale. 


\section{References}

1. Cheng MZ, Zaman G, Lanyon LE. Estrogen enhances the stimulation of bone collagen synthesis by loading and exogenous prostacyclin, but not prostaglandin E2, in organ cultures of rat ulnae. J Bone Miner Res. 1994;9(6):805-816.

2. Rawlinson SC, el-Haj AJ, Minter SL, Tavares IA, Bennett A, Lanyon LE. Loadingrelated increases in prostaglandin production in cores of adult canine cancellous bone in vitro: a role for prostacyclin in adaptive bone remodeling? J Bone Miner Res. 1991; 6(12): 1345-1351.

3. Severson AR, Rothberg PF, Pratt RM, Goggins JF. Effect of parathyroid hormone on the incorporation of $3 \mathrm{H}$-glucosamine into hyaluronic acid in bone organ culture. Endocrinology. Apr 1973; 92(4): 1282-1285.

4. Kaji T, Kawatani R, Hoshino $T$, et al. A suitable culture medium for ossification of embryonic chick femur in organ culture. Bone Miner. 1990; 9(2):89-100.

5. Van Loon JJ, Bervoets DJ, Burger EH, et al. Decreased mineralization and increased calcium release in isolated fetal mouse long bones under near weightlessness. J Bone Miner Res. 1995; 10(4):550-557.

6. el Haj AJ, Minter SL, Rawlinson SC, Suswillo R, Lanyon LE. Cellular responses to mechanical loading in vitro. J Bone Miner Res. 1990;5(9):923-932.

7. Takai E, Mauck RL, Hung CT, Guo XE. Osteocyte viability and regulation of osteoblast function in a 3D trabecular bone explant under dynamic hydrostatic pressure. J Bone Miner Res. 2004; 19(9): 1403-1410.

8. Jones DB, Broeckmann E, Pohl T, Smith EL. Development of a mechanical testing and loading system for trabecular bone studies for long term culture. Eur Cell Mater. 2003; 5: 48-59; discussion 59-60.

9. Mann V, Huber C, Kogianni G, Jones D, Noble B. The influence of mechanical stimulation on osteocyte apoptosis and bone viability in human trabecular bone. J Musculoskelet Neuronal Interact. 2006;6(4):408-417.

10. Liebschner MA. Biomechanical considerations of animal models used in tissue engineering of bone. Biomaterials. 2004; 25(9): 1697-1714.

11. Davies CM, Jones DB, Stoddart MJ, et al. Mechanically loaded ex vivo bone culture system 'Zetos': systems and culture preparation. Eur Cell Mater. 2006; 11:57-75; discussion 75.

12. McNair PJ, Prapavessis $\mathrm{H}$. Normative data of vertical ground reaction forces during landing from a jump. J Sci Med Sport. 1999;2(1):86-88.

13. Hildebrand $T$, Ruegsegger P. A new method for the model independent assessment of thickness in three-dimensional images. J Microsc. 1997; 185:67-75.

14. Hildebrand T, Ruegsegger P. Quantification of Bone Microarchitecture with the Structure Model Index. Comput Methods Biomech Biomed Engin. 1997; 1(1):1523.

15. Whitehouse WJ. The quantitative morphology of anisotropic trabecular bone. J Microsc. 1974; 101 Pt 2:153-168.

16. Odgaard A, Gundersen HJ. Quantification of connectivity in cancellous bone, with special emphasis on 3-D reconstructions. Bone. 1993; 14(2): 173-182.

17. David V, Laroche N, Boudignon B, et al. Noninvasive in vivo monitoring of bone architecture alterations in hindlimb-unloaded female rats using novel threedimensional microcomputed tomography. J Bone Miner Res. Sep 2003; 18(9): 1622-1631.

18. Martin A, de Vittoris R, David V, et al. Leptin modulates both resorption and formation while preventing disuse-induced bone loss in tail-suspended female rats. Endocrinology. 2005; 146(8):3652-3659.

19. Noble BS, Stevens HY. Techniques for the study of apoptosis in bone. Methods Mol Med. 2003; 80:225-236.

20. Un K, Bevill G, Keaveny TM. The effects of side-artifacts on the elastic modulus of trabecular bone. J Biomech. 2006; 39(11):1955-1963.

21. Harrigan TP, Jasty M, Mann RW, Harris WH. Limitations of the continuum 
assumption in cancellous bone. J Biomech. 1988; 21(4):269-275.

22. Lian JB, Stein GS. Concepts of osteoblast growth and differentiation: basis for modulation of bone cell development and tissue formation. Crit Rev Oral Biol Med. 1992; 3(3):269-305.

23. Lian JB, Stein GS. The developmental stages of osteoblast growth and differentiation exhibit selective responses of genes to growth factors (TGF beta 1) and hormones (vitamin D and glucocorticoids). J Oral Implantol. 1993;19(2): 95105; discussion 136-107.

24. Barou O, Lafage-Proust $\mathrm{MH}$, Martel $\mathrm{C}$, et al. Bisphosphonate effects in rat unloaded hindlimb bone loss model: three-dimensional microcomputed tomographic, histomorphometric, and densitometric analyses. J Pharmacol Exp Ther. 1999; 291(1):321-328.

25. Stauber M, Muller R. Age-related changes in trabecular bone microstructures: global and local morphometry. Osteoporos Int. 2006; 17(4):616-626.

26. Parfitt AM. Osteonal and hemi-osteonal remodeling: the spatial and temporal framework for signal traffic in adult human bone. J Cell Biochem. 1994; 55(3): 273-286.

27. Yao Z, Lafage-Proust MH, Plouet J, Bloomfield S, Alexandre C, Vico L. Increase of both angiogenesis and bone mass in response to exercise depends on VEGF. J Bone Miner Res. 2004; 19(9): 1471-1480.

28. Mori T, Okimoto N, Sakai A, et al. Climbing exercise increases bone mass and trabecular bone turnover through transient regulation of marrow osteogenic and osteoclastogenic potentials in mice. J Bone Miner Res. 2003; 18(11):2002-2009.

29. Creighton DL, Morgan AL, Boardley D, Brolinson PG. Weight-bearing exercise and markers of bone turnover in female athletes. J Appl Physiol. 2001; 90(2):565570. 


\section{Figure Legends}

Figure 1. 3D dynamic bone culture in the ZetOS $^{T M}$ bioreactor - technical set up

(A) The ZetOS ${ }^{\mathrm{TM}}$ apparatus (described extensively in Jones et al., $2003^{8}$ ) includes a loading device (left), a culture chamber positioned in the lower part of the loading device, a peristaltic pump (medium) and an individual culture medium tanks (right). Twenty four culture chambers can be installed at the same time; (B) Detail of the culture chamber where the sample will be fitted, inner diameter: $1 \mathrm{~cm}$. Inlet and outlet for medium culture circulation. After installation of the sample, a sapphire piston will close the chamber. (AB) Bars: $1 \mathrm{~cm}$; (C) 3D microCT-scan of a sternum sample, bar: $250 \mu \mathrm{m}$; (D) Visualization (arrows) of the lamellar organization of bovine cancellous bone in a sternum sample observed under polarized light; (E) Tetracycline double labelings (arrows) of mineralizing surfaces of a sternum trabecula after 3 weeks of culture. (F) Modified Goldner staining showing mineralized tissue and osteoid seam. Note that the marrow is mainly hematopoietic with only few adipocytes; D-F; Bars: $1 \mathrm{~mm}$.

\section{Figure 2. Histology and long term sample viability.}

(A-B) Histochemical reaction for LDH enzyme in cryostat sections. Sternum biopsies after 3 weeks of culture. Viable osteocytes (A) stained darkly for LDH activity. To distinguish viable osteocytes from dead ones, DAPI nuclear staining was performed on LDH-treated sections (B); arrows: nonviable cells stained for DAPI but not LDH. (C) Percent of viable osteocytes and (D) Bone forming rate assessed in ulna and sternum bone cores after 3 weeks for control (C) or loaded (L) cultures. Values are mean \pm SE of 8 samples per group. *: $p<0.05$ vs matched $C$ samples. Bars: $1 \mathrm{~mm}$

\section{Figure 3. Effects of cyclic mechanical loading on bone formation.}

(A) Alkaline phosphatase activity measured in culture media of control (dark squares) or loaded (open circles) samples collected over 3 weeks of culture. (B) Alkaline phosphatase activity measured in tissue lysates of control (open bars) or loaded (gray bars) samples harvested at 7, 14 and 21 days of culture. (C) Runx2 protein amounts and (D) gene 
expression. (E) Osteocalcin protein amounts and (F) gene expression were assessed in cell lysates. Protein values were normalized on total protein content and expressed as percent of control at day 7; mRNA values were normalized on the housekeeping gene L30 and expressed as percent of basal control expression. Values are mean \pm SE of 8 samples per group. $p<0.05$ vs *: C, \#: day $7, \$$ : day 14 .

\section{Figure 4. Effects of cyclic mechanical loading on bone resorption.}

(A) Time course assessment of type I collagen C-telopeptide (CTX) concentrations in culture media of control (dark squares) and loaded (open circles) samples during 3 weeks. Values are mean \pm SE of 8 samples per group. (B-C) Loaded bone cores were either left untreated (open bars), or treated with $10^{-8} \mathrm{M}$ retinoic acid (RA) during the first 3 days of culture (dark bars), or treated with retinoic acid as described, then with $10^{-6} \mathrm{M}$ pamidronate during the next 3 days (gray bars). Starting at day $6, \mathrm{Ca}^{2+}(\mathbf{B})$ and CTX (C) levels were assayed in the culture medium. Values are mean \pm SE of 4 samples per group. *: $p<0.05$ vs untreated, $\# p<0.05$ vs RA.

Figure 5. Evolution of bone structure in loaded and unloaded bone core samples cultured in the Zetos ${ }^{\mathrm{TM}}$ bioreactor.

(A) Longitudinal study of trabecular thickness (Tb.Th) and (B) structural model index (SMI) in controls (open circles; doted lines) and loaded (black circles; bolded lines) samples cultured during 3 weeks. Values are mean \pm SE of 8 samples per group: \#: $p<0.05$ vs control at d0,*: $p<0.05$ vs matched control at day 21 . (C) Young's modulus and (D) Ultimate Force of bone core samples either untreated (Basal Controls: BC), perfused (C) or perfused and loaded (L) for 3 weeks. *: $p<0.05$ vs $C, \#:<0.05, \# \# \#$ : $<0.001$ vs $B C, N=8$. 


\section{Tables}

Table 1. Histomorphometry data for bone cell activities.

\begin{tabular}{|c|c|c|c|}
\hline Parameters & Basal Control & $\begin{array}{l}\text { Control } \\
\text { (Perfused) }\end{array}$ & $\begin{array}{c}\text { Loaded } \\
\text { (Perfused \& Loaded) }\end{array}$ \\
\hline \multicolumn{4}{|l|}{ Bone formation } \\
\hline OS/BS (\%) & $7.05 \pm 1.53$ & $9.72 \pm 0.94$ & $14.37 \pm 1.16(*)$ \\
\hline O.Th $(\mu \mathrm{m})$ & $10.61 \pm 1.19$ & $10.38 \pm 0.90$ & $12.6 \pm 1.17(*, \#)$ \\
\hline MAR $\left(\mu \mathrm{m} /\right.$ day $\left.^{-1}\right)$ & & $0.71 \pm 0.05$ & $0.94 \pm 0.05(\#)$ \\
\hline BFR/BS $\left(\mu \mathrm{m}^{3} / \mu \mathrm{m}^{2} /\right.$ day $)$ & & $0.0079 \pm 0.0010$ & $0.0116 \pm 0.0014(\#)$ \\
\hline \multicolumn{4}{|l|}{ Bone resorption } \\
\hline Oc.S/BS (\%) & $4.03 \pm 0.22$ & $3.06 \pm 0.37$ & $3.12 \pm 0.35$ \\
\hline Density of & $12.08 \pm 0.76$ & $7.35 \pm 0.93(*)$ & $8.52 \pm 0.95(*)$ \\
\hline Resorption Lacunae $\left(\mathrm{mm}^{-2}\right)$ & & & \\
\hline
\end{tabular}

Table 2. 3D Microarchitectural and mechanical parameters.

\begin{tabular}{|c|c|c|c|}
\hline Parameters & Basal Control & $\begin{array}{l}\text { Control } \\
\text { (Perfused) }\end{array}$ & $\begin{array}{c}\text { Loaded } \\
\text { (Perfused \& Loaded) }\end{array}$ \\
\hline
\end{tabular}

\section{Mass \& Density}

VOX-BV/TV (\%)

$11.16 \pm 0.82$

$10.73 \pm 0.80$

$13.9 \pm 0.79$

Lin. Att. 2

$1.95 \pm 0.003$

$1.95 \pm 0.006$

$1.94 \pm 0.009$

\section{Architecture}

\begin{tabular}{llll} 
Tb Th $(\mu \mathrm{m})$ & $84.30 \pm 5.00$ & $84.5 \pm 3.19$ & $104.2 \pm 5.09(*, \#)$ \\
SMI & $1.24 \pm 0.06$ & $1.38 \pm 0.07$ & $0.87 \pm 0.07(*, \#)$ \\
DA & $1.58 \pm 0.003$ & $1.45 \pm 0.05$ & $1.64 \pm 0.10(*)$ \\
Conn-Dens. $\left(\mathrm{mm}^{-1}\right)$ & $12.59 \pm 1.44$ & $12.12 \pm 0.86$ & $8.79 \pm 0.57(*, \#)$ \\
\hline
\end{tabular}

Values are mean \pm SEM of $n=10$ samples per group; $p<0.05\left(^{*}\right)$ vs. Basal Control, (\#) vs. Control. 

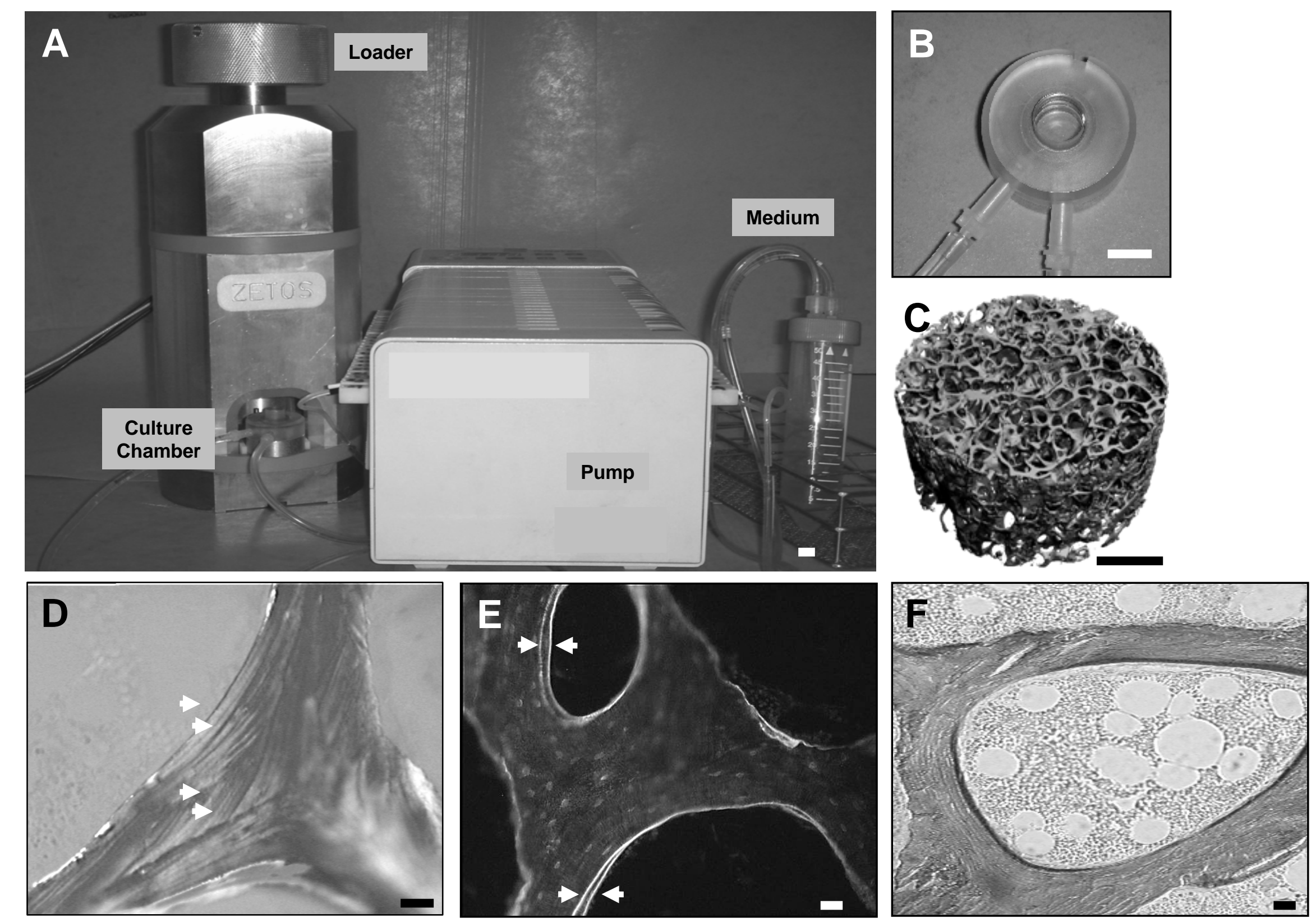

David et al., 2006 - Figure 1 

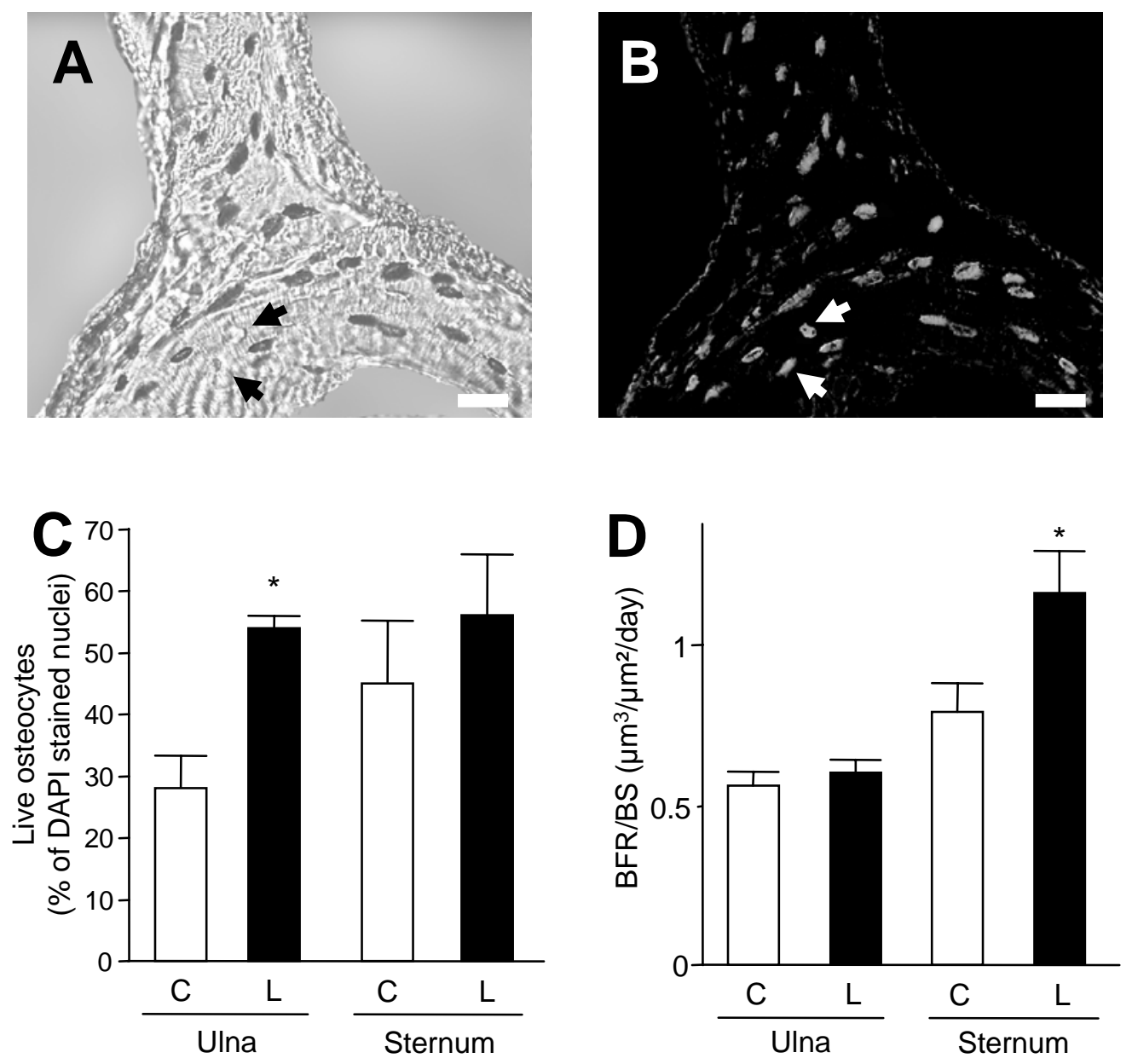

David et al., 2006 - Figure 2 

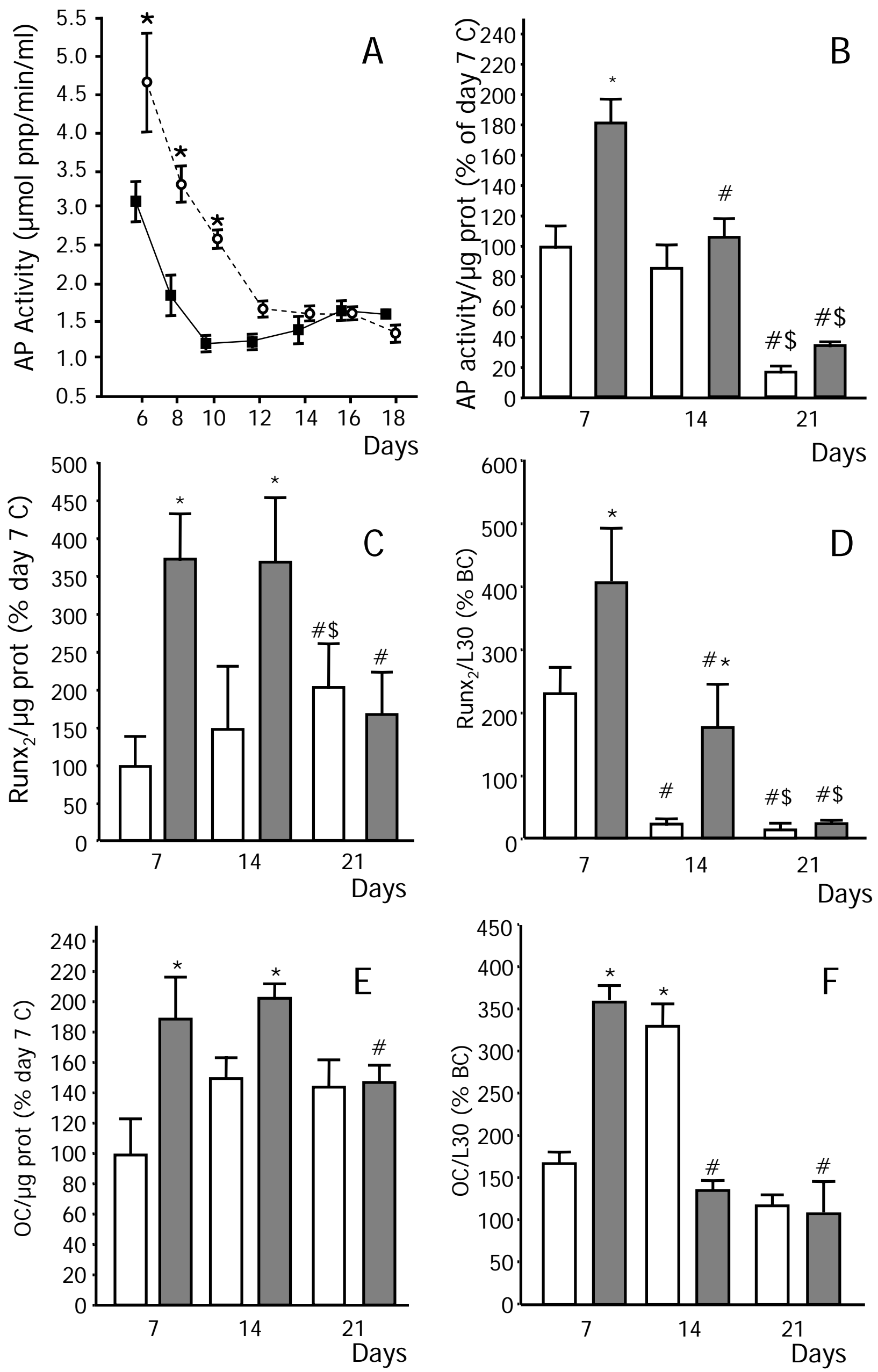

David et al., 2006 - Figure 3 

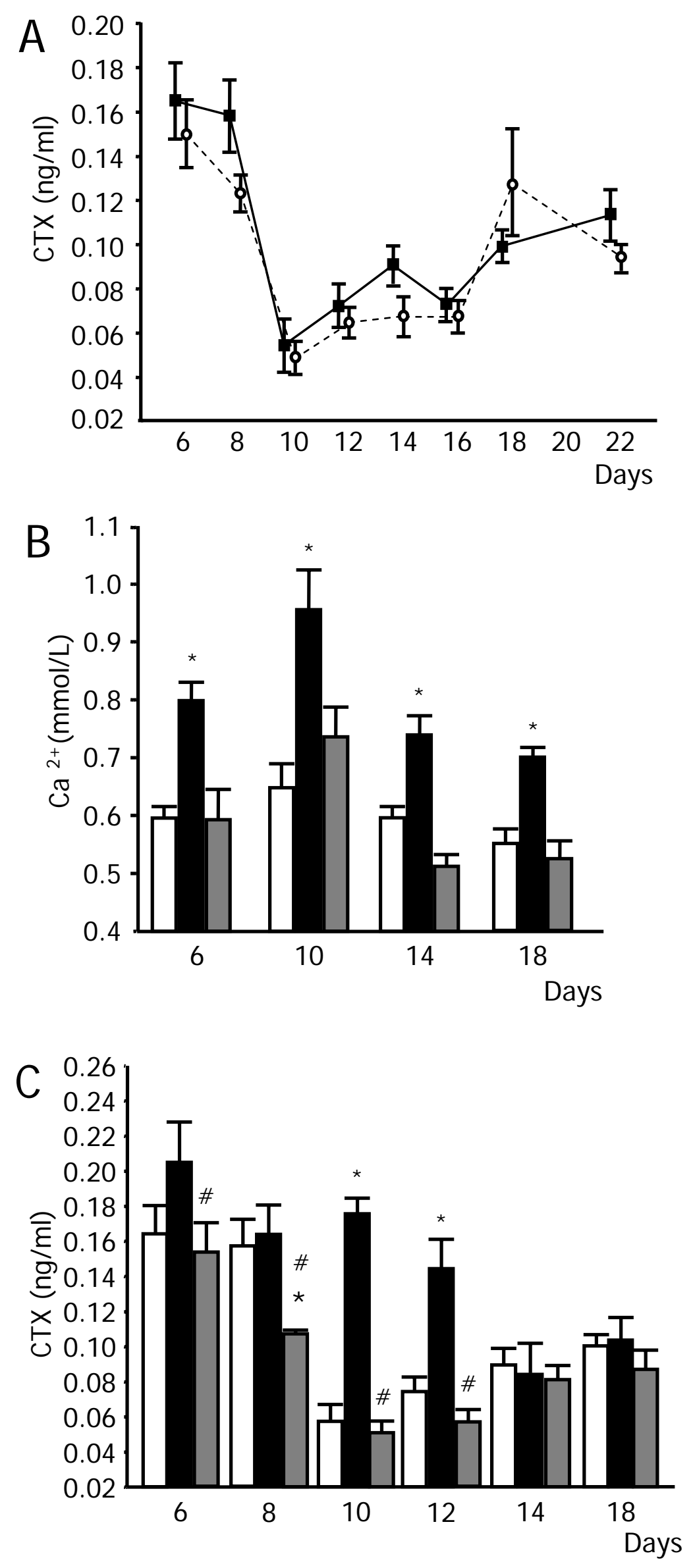

David et al., 2006 - Figure 4 

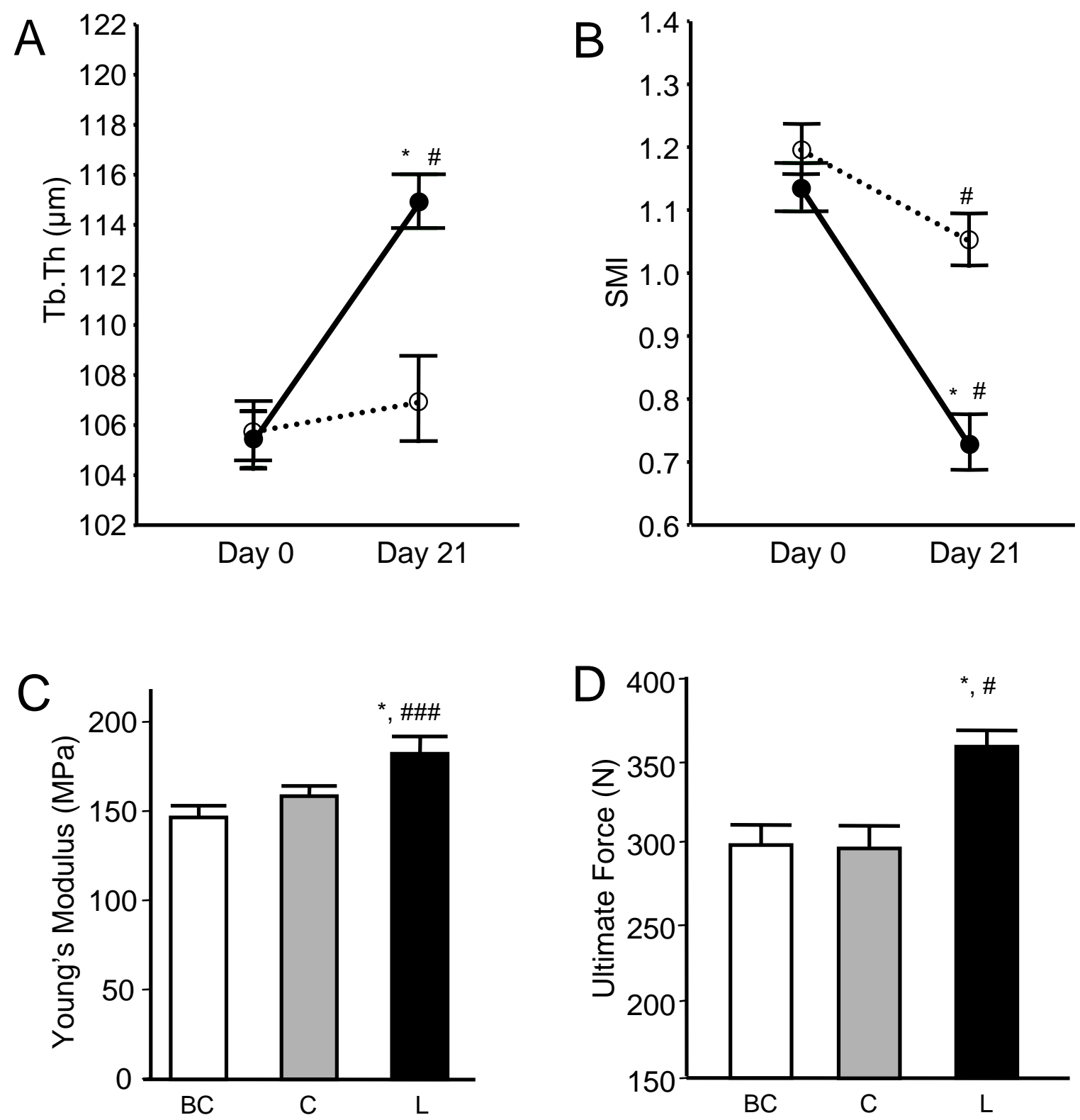

David et al., 2006 - Figure 5 